\title{
Iterative Maximal Ratio Combining Channel Estimation for Multiuser Detection on a Time Frequency Selective Wireless CDMA Channel
}

\author{
Zhenning Shi Mark C. Reed \\ Wireless Signal Processing Program, National ICT Australia \\ Research School of Information Sciences and Engineering, Australian National University \\ Braddon, ACT 2612, Australia \\ Email: $\{$ zhenning.shi, mark.reed $\}$ @nicta.com.au
}

\begin{abstract}
In this paper, we investigate the performance of DS-CDMA systems in dispersive fading channels. We develop a low-complexity receiver that jointly performs channel estimation and multiuser decoding in an iterative manner. Pilot symbols, together with the soft decoding information on the transmitted data are harnessed to estimate the time-varying channel states, which in turn leads to improved detection and decoding results. We show through simulation and mean square error analysis that the proposed system can sustain a high-throughput WCDMA transmission.
\end{abstract}

\section{INTRODUCTION}

Code-division multiple-access (CDMA) is a communication system where a number of users can simultaneously access the common channel. Forward link CDMA separates different users with orthogonal spreading sequences. However, return link WCDMA [1] system allows random codes, which destroys the orthogonality between users and causes severe multiple-access interference (MAI). Multiuser detection [8], [12] has been studied to address MAI to achieve good system performance, at the cost of increasing the complexity at the receiver.

CDMA transmission over a time-varying multipath channel suffers a degradation on signal quality from the inter-symbol interference (ISI). Either pilots or training symbols are used for channel estimation and equalization in common practice. However, in a multi-user scenario, channel estimation is subject to MAI and has significant estimation errors, which would be carried over into detection and decoding in the later stage.

Choi proposes a method that combines pilot as well as traffic channel information in joint channel estimation and data detection in [6]. To avoid large delay, an adaptive algorithm is developed and shown to outperform pilot-aided method in single-user scenario. A decision-directed method is assumed by Bhashyam and Aazhang in [7] to estimate channel parameters following the gradient descent and steepest descent approaches. To that end, correlation matrix for spread data has to be computed each time the channel estimates are updated. The use of hard decoding information in tracking channel may introduce error propagation.

Since the advent of turbo coding, iterative processing principle has found applications in many communication regimes.
Iterative multiuser detection has been shown to achieve very high throughput communications for heavily loaded CDMA. However, most state-of-art designs [9]-[11] assume that perfect channel state information (CSI) is known at the receiver. To enable the iterative receivers to work properly in realistic scenarios, channel parameter estimation has to be taken into account in the system design. In [5], Otnes and Tüchler investigate on iterative channel estimation for single-user timevarying frequency selective channels. They separate channel estimation from the equalization and incorporate the fed back information from the decoder to improve the channel estimates over the iterations. Channel estimation for multiuser channels is more challenging due to the presence of MAI, and the interference cancellation is found to be sensitive to errors in channel parameter estimates. In [2], Alexander and Grant develop an iterative receiver for CDMA that reconstructs and cancels the MAI using the soft information from decoders and channel estimates. The improved signals after the interference cancellation are in turn used to enhance the performance of channel estimation. To increase the robustness of the system, damping and partial interference cancellation are proposed for a multistage receiver in [3] to alleviate error propagation. Lampe apply MMSE criterion to channel estimation and achieve near-optimal performance over iterations [4].

In this paper, we propose a novel iterative receiver for WCDMA systems where channel estimation, interference cancellation and channel decoding are performed interchangeably to enhance the estimation/decoding quality. Unlike other iterative systems, we use both pilot and soft decoding information for channel estimation, and combine the two channel estimates by weights that are adjusted according to the statistics used. We analyze the mean square error (MSE) on estimated parameters for the new system. The MSE analysis, together with the BER performance of the new system, is shown to perform very well in a variety of multipath channels.

The paper is organized as follows. In Section II, the system model for WCDMA uplink channel is introduced. An iterative receiver for joint channel estimation and data decoding is outlined in Section III. A Maximal-ratio combining (MRC) based channel estimation method is proposed in Section IV, and 
its MSE is analyzed in Section V. In Section VI, simulation results are reported, and main conclusions are summarized in Section VII.

\section{System Model for Uplink WCDMA}

We consider the uplink of a $K$-user DS-CDMA system that allows asynchronous transmission. Denote by $d_{k, m}$ the $m$-th coded bit for user $k, s_{k, d}(t)$ the spreading sequence for user $k$ 's data, $c_{k, m}$ the $m$-th pilot bits for user $k$ and $s_{k, c}(t)$ the spreading sequence for user $k$ 's pilots, the spread signal is given by

$$
\begin{array}{r}
x_{k}(t)=\sum_{m=0}^{M-1} \sqrt{E_{d, k}} d_{k, m} s_{k, d}(t-m T) \\
+j \sqrt{E_{p, k}} c_{k, m} s_{k, c}(t-m T)
\end{array}
$$

where the first term in (1) is for DPDCH channel of user $k$, the second term is for the orthogonal DPCCH traffic, $T$ is the time interval for one symbol, and $E_{d, k}, E_{p, k}$ are the powers for data and pilots respectively. We focus on equal power transmission, i.e., $E_{d, k} \equiv E_{d}, E_{p, k} \equiv E_{p}, \forall k$, hence the user index will be dropped in later discussions.

The transmission between the transmit and receive ends is over a multipath fading channel that is characterized by the time-varying parameters. We use a tapped-delay-line channel model to represent the $L$-path channel for user $k$, with path gains $h_{k, 0}(t), h_{k, 1}(t), \ldots, h_{k, L-1}(t)$. The path gains are assumed to be independent and identically distributed complex Gaussian processes with the autocorrelation satisfying the zero-order Bessel function $J_{0}\left(2 \pi f_{d} \tau\right)$ of the first kind, where $f_{d}$ is the maximal doppler frequency.

The received signal at the base station is then given by

$$
r(t)=\sum_{k=1}^{K} \sum_{l=0}^{L-1} h_{k, l}(t) x_{k}\left(t-\tau_{k, l}\right)+n(t)
$$

where $\tau_{k, l}$ is the delay for user $k$ 's $l$-th path and $n(t)$ is the complex Gaussian noise with variance $N_{0}$.

\section{Iterative Multiuser Receiver}

A coherent receiver that operates in the turbo fashion is shown in Figure 1. Two main soft-input soft-output (SISO) modules exchange the extrinsic information on user data $d_{k, m}$ and improve its reliability over the iterations. Assume perfect timing information is available, the MAI and ISI term for $l$-th path of user $k$ can be re-constructed using the soft information from the decoder, given by (3). In (3), $\hat{h}_{i, q}$ is the estimate for user $i$ 's $q$-th path gain and $\tilde{d}_{k, m}=\tanh \left(\lambda_{k, m}^{(E)} / 2\right)$ is the bit estimate based on the extrinsic decoding LLR.

In the MAI/ISI cancellation module, received signals subtract the re-constructed MAI/ISI and matched filter with the spreading sequence to obtain the partial signal for DPDCH and DPCCH channel as

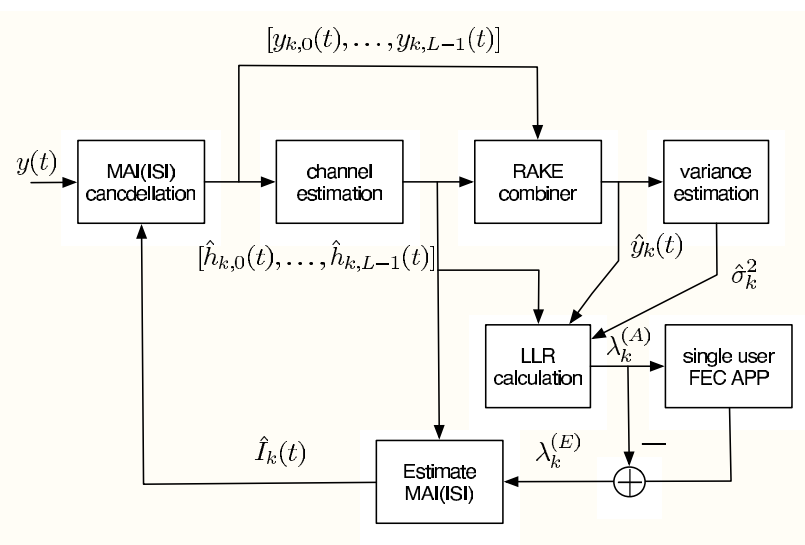

Fig. 1. An Iterative receiver for DS-CDMA

$$
\begin{aligned}
& y_{k, l, d}(t)=\left(r(t)-\hat{I}_{k, l}(t)\right) \otimes s_{k, d}\left(-t+\tau_{k, l}\right) \\
& y_{k, l, c}(t)=\left(r(t)-\hat{I}_{k, l}(t)\right) \otimes s_{k, c}\left(-t+\tau_{k, l}\right)
\end{aligned}
$$

In (4) and (5), $\otimes$ represents convolution. Both $y_{k, l, d}$ and $y_{k, l, c}$ are used to estimate the time-varying path gain $h_{k, l}$ in the channel estimation module, using the methods to be discussed in the next section. RAKE module is to combine all the partial data signals $\left\{y_{k, l, d}\right\}_{l=0}^{L-1}$ from the delayed paths to form enhanced observation values, as for user $k$, given by

$$
y_{k}(m)=\sum_{l=0}^{L-1} \frac{\hat{h}_{k, l}^{*}(m)}{\sqrt{\sum_{l}\left\|\hat{h}_{k, l}(m)\right\|^{2}}} y_{k, l, d}\left(m T+\tau_{k, l}\right)
$$

where $\hat{h}_{k, l}(m)$ is $l$-th the path gain estimate for user $k$ for the $m$-th bit. Channel fading effect is compensated at this stage by making use of the multipath diversity to provide a reliable statistics, which we assume to satisfy an AWGN model, i.e., $y_{k}(m)=\sqrt{E_{d} \sum_{l=0}^{L-1}\left\|\hat{h}_{k, l}(m)\right\|^{2}} d_{k, m}+n_{k}(m)$, where $n_{k}(m) \sim \mathcal{C N}\left(0, \sigma_{k}^{2}\right)$ is the residual interference-plus-noise embedded in $y_{k}(m)$ with $\sigma_{k}^{2}$ being the variance. The input LLR for APP channel decoder is then calculated as

$$
\begin{aligned}
\lambda_{k}^{(A)}(m) & =\log \left(\frac{P\left(d_{k, m}=1 \mid y_{k}(m),\left\{\hat{h}_{k, l}(m)\right\}_{l=0}^{L-1}\right)}{P\left(d_{k, m}=-1 \mid y_{k}(m),\left\{\hat{h}_{k, l}(m)\right\}_{l=0}^{L-1}\right)}\right) \\
& =\frac{4 \sqrt{E_{d}}}{\hat{\sigma}_{k}^{2}(m)} \sqrt{\sum_{l=0}^{L-1}\left\|\hat{h}_{k, l}(m)\right\|^{2}} y_{k}(m)
\end{aligned}
$$

In (7), the online estimate of the residual noise variance is denoted by $\hat{\sigma}_{k}^{2}(m)$. Several data-aided (DA) and non-dataaided (NDA) variance estimation method have been studied in [13]-[15]. In this paper, we apply a simple NDA algorithm, where the variance is estimated from the RAKE outputs within a moving-average-window (MAW) as 


$$
\begin{aligned}
\hat{I}_{k, l}(t)= & \overbrace{\sum_{i \neq k} \sum_{q=0}^{L-1} \sum_{m=0}^{M-1} \hat{h}_{i, q}(t-m T)\left[\sqrt{E_{d}} \tilde{d}_{i, m} s_{i, d}\left(t-m T-\tau_{i, q}\right)+j \sqrt{E_{p}} c_{i, m} s_{i, c}\left(t-m T-\tau_{i, q}\right)\right.}^{\text {MAI }} \\
& +\underbrace{\sum_{q \neq l} \sum_{m=0}^{M-1} \hat{h}_{k, q}(t-m T)\left[\sqrt{E_{d}} \tilde{d}_{k, m} s_{k, d}\left(t-m T-\tau_{k, q}\right)+j \sqrt{E_{p}} c_{k, m} s_{k, c}\left(t-m T-\tau_{k, q}\right)\right.}_{\text {ISI }}]
\end{aligned}
$$

$$
\begin{aligned}
\hat{\sigma}_{k}^{2}(m)=E & {\left[\left\|y_{k}\right\|^{2}\right]-E^{2}\left[y_{k} \mid d_{k}\right] \approx \frac{1}{2 N+1} \sum_{i=m-N}^{m+N}\left\|y_{k}(i)\right\|^{2} } \\
& -\left(\frac{1}{2 N+1} \sum_{i=m-N}^{m+N}\left|y_{k}(i)\right|\right)^{2}
\end{aligned}
$$

where $2 N+1$ is the length of the MAW. We observe that this variance estimation approach is robust in a multipath scenario, and the whole system performance is not noticeably different from that employing the more advanced SNR estimation techniques.

Single user FEC decoders take as input the a priori LLR $\lambda_{k}^{(A)}$ and perform the APP decoding. The output extrinsic information $\lambda_{k}^{(E)}$ is then used in the next iteration to reestimate the channel and MAI/ISI.

\section{MRC-BASED CHANNEL Estimation}

As discussed in the previous section, channel state information (CSI) is required in re-constructing the MAI/ISI for interference cancellation, RAKE combining and LLR computation. Hence the knowledge of CSI is crucial to the effectiveness of the iterative receiver in Figure 1. In [3], it is reported that inaccurate CSI estimates leads to the failure of coherent CDMA reception.

In this section, we derive a new maximal-ratio-combining (MRC)-based channel estimation algorithm, which is based on the coarse estimates from the basic pilot-aided and data-aided methods. In [2], CDMA systems that use the soft decoding data as the reference signals to estimate the channel are reported. The unconstrained method computes the path gain for $l$-th path of user $k$ as

$$
\hat{h}_{k, l, d}(m)=y_{k, l, d}\left(m T+\tau_{k, l}\right) \frac{\tilde{d}_{k, m}}{\sqrt{E_{d}}}
$$

The sub-index $d$ in (9) indicates that the so obtained CSI knowledge is based on the traffic data. This approach is limited by the available information on the transmitted data, especially in the first couple of iterations when the soft symbol estimates are trivial. Another estimation method is to infer the channel parameters with the aid of pilot symbols as

$$
\hat{h}_{k, l, c}(m)=y_{k, l, c}\left(m T+\tau_{k, l}\right) \frac{c_{k, m}}{\sqrt{E_{p}}}
$$

Pilot-aided methods do not suffer from the degeneration on the reference signals. However, the low power allocated to the pilot symbols leads to an unsatisfying performance for channel estimation.

In this paper, we apply the CSI knowledge from both the pilot and soft decoding data in channel estimation. A heuristic approach is to use the mean of the two estimated values as $\hat{h}_{k, l}(m)=\frac{1}{2}\left(\hat{h}_{k, l, d}(m)+\hat{h}_{k, l, c}(m)\right)$, which we name as the arithmetic average (AA) channel estimation. AA estimation does not seek to combine the CSI information from two sources, i.e., pilots and data, in a judicious way, hence it causes performance degeneration especially when the CSI estimates from one source is significantly inferior to the other. We assume that the unconstrained CSI estimates from (9) and (10) conform to the following model

$$
\begin{aligned}
& \hat{h}_{k, l, d}(m)=\sqrt{\left\|\tilde{d}_{k}\right\|^{2}} h_{k, l}+n_{l, d} \\
& \hat{h}_{k, l, c}(m)=h_{k, l}+n_{l, c}
\end{aligned}
$$

where $n_{l, d}$ and $n_{l, c}$ are estimation errors that are complex Gaussian. The variances of the two are related as $E\left[\left\|n_{l, d}\right\|^{2}\right.$ ]$/ E\left[\left\|n_{l, c}\right\|^{2}\right]=\left(E_{p}\left\|\tilde{d}_{k}\right\|^{2}\right) /\left(E_{d} \gamma\right)$, with $\gamma$ being the ratio of spreading gains of the two multiplexed channels.

To find a CSI estimate that has the least mean square error (MSE), maximal ratio combining (MRC) can be used to derive the optimal weights. A Lagrange problem can be formulated according to the MRC rule as

$$
\begin{aligned}
\hat{h}_{k, l}= & w_{1} \hat{h}_{k, l, c}+w_{2} \hat{h}_{k, l, d} \\
= & \left(w_{1}+w_{2} \sqrt{\left\|\tilde{d}_{k}\right\|^{2}}\right) h_{k, l}+\left(w_{1} n_{k, l, d}+w_{2} n_{k, l, c}\right) \\
\mathbf{w}_{\mathrm{opt}}= & \arg \min _{w_{1}, w_{2}}\left(w_{1}^{2}+w_{2}^{2} \frac{E_{p}}{E_{d} \gamma}\right) \sigma_{l}^{2} \\
& +\eta\left(w_{1}+w_{2} \sqrt{\left\|\tilde{d}_{k}\right\|^{2}}-1\right)
\end{aligned}
$$

where $\sigma_{l}^{2}=E\left[\left\|n_{k, l, c}\right\|^{2}\right]$ is the power for pilot channel estimates and $\eta$ is the Lagrange multiplier. Solving (13), we get the optimal weights, given by 


$$
\begin{aligned}
w_{1, \mathrm{opt}} & =\frac{1}{\frac{E_{d}}{E_{p}} \gamma+1}, \\
w_{2, \mathrm{opt}} & =\frac{\frac{E_{d}}{E_{p}} \gamma}{\sqrt{\left\|\tilde{d}_{k}\right\|^{2}}\left(\frac{E_{d}}{E_{p}} \gamma+1\right)}
\end{aligned}
$$

where $\left\|\tilde{d}_{k}\right\|^{2}=E\left[d_{k}^{2} \mid \tilde{d}_{k}\right]$ is the power for the conjectured soft symbols to represent the reliability of the fed back information. This parameter can be approximated as the average of online values as $\left\|\tilde{d}_{k}(m)\right\|^{2} \approx \frac{1}{2 Q-1} \sum_{i=m-Q}^{m+Q} \tilde{d}_{i}^{2}$.

\section{MSE ANALYSIS}

MRC-based algorithm combines the CSI knowledge from both channels in an optimal way by adjusting the weights according to the reliability level of the soft decoding information. To quantitize its performance in comparison to that of the other three channel estimation methods, we derive the $\mathrm{MSE}^{1}$ of the output channel estimates for them.

It can be shown that the MSEs are given by

$$
\begin{aligned}
\operatorname{MSE}_{l}^{(\mathrm{PA})} & =\frac{\sigma_{l}^{2}}{\left\|h_{l}\right\|^{2}} \\
\mathrm{MSE}_{l}^{(\mathrm{DA})} & =\frac{E_{p} \sigma_{l}^{2}}{\gamma E_{d}\left\|h_{l}\right\|^{2}} \\
\mathrm{MSE}_{l}^{(\mathrm{AA})} & =\left(\frac{1+\frac{E_{p}\left\|\tilde{d}_{k}\right\|^{2}}{E_{d} \gamma}}{\left(1+\sqrt{\left\|\tilde{d}_{k}\right\|^{2}}\right)^{2}}\right) \frac{\sigma_{l}^{2}}{\left\|h_{l}\right\|^{2}}
\end{aligned}
$$

for (10), (9) and AA methods, respectively, where $\left\|h_{l}\right\|^{2}$ is the channel power of path $l$. Using the optimal weights in (15), MSE of the MRC-based algorithm can be derived as

$$
\mathrm{MSE}_{l}^{(\mathrm{MRC})}=\left(\frac{1}{1+\frac{E_{d} \gamma}{E_{p}}}\right) \frac{\sigma_{l}^{2}}{\left\|h_{l}\right\|^{2}}
$$

The improvement of the MRC method over others can be inferred from the ratio between $\mathrm{MSE}_{l}^{(\mathrm{MRC})}$ and those in (18), which are given by

$$
\begin{aligned}
& \beta_{l}^{(\mathrm{PA})} \triangleq \frac{\mathrm{MSE}_{l}^{(\mathrm{PA})}}{\mathrm{MSE}_{l}^{(\mathrm{MRC})}}=1+\frac{E_{d} \gamma}{E_{p}} \\
& \beta_{l}^{(\mathrm{DA})} \triangleq \frac{\mathrm{MSE}_{l}^{(\mathrm{DA})}}{\mathrm{MSE}_{l}^{(\mathrm{MRC})}}=1+\frac{E_{p}}{E_{d} \gamma} \\
& \beta_{l}^{(\mathrm{AA})} \triangleq \frac{\mathrm{MSE}_{l}^{(\mathrm{AA})}}{\mathrm{MSE}_{l}^{(\mathrm{MRC})}}=\frac{\left(1+\frac{E_{d} \gamma}{E_{p}}\right)\left(1+\frac{E_{p}\left\|\tilde{d}_{k}\right\|^{2}}{E_{d} \gamma}\right)}{\left(1+\sqrt{\left\|\tilde{d}_{k}\right\|^{2}}\right)^{2}}
\end{aligned}
$$

for PA, DA and AA channel estimators, respectively. It is straightforward to show from (20) and (21) that MRC-based method outperforms PA and DA algorithms. To show that

\footnotetext{
${ }^{1}$ The mean square error we consider here is the error variance normalized w.r.t. the signal power
}

MRC is superior to AA method, we note that $\gamma=\frac{N_{p}}{N_{d}}, E_{d}=$ $E_{d, c} N_{d}$ and $E_{p}=E_{p, c} N_{p}$, where $N_{p}, N_{d}$ are the spreading gains for DPCCH and DPDCH channels, and $E_{p, c}, E_{d, c}$ are power within one chip duration for the two multiplexed channels. Equation (22) then becomes

$$
\beta_{l}^{(\mathrm{AA})}=\frac{\left(1+\frac{E_{d, c}}{E_{p, c}}\right)\left(1+\frac{E_{p, c}\left\|\tilde{d}_{k}\right\|^{2}}{E_{d, c}}\right)}{\left(1+\sqrt{\left\|\tilde{d}_{k}\right\|^{2}}\right)^{2}}
$$

Take the derivative of $\beta_{l}^{(\mathrm{AA})}$ w.r.t. $\|\tilde{d}\|^{2}$, we get

$$
\frac{\partial \beta_{l}^{(\mathrm{AA})}}{\partial\left\|\tilde{d}_{k}\right\|^{2}}=\frac{\left(1+\frac{E_{d, c}}{E_{p, c}}\right)\left(\frac{E_{p, c}}{E_{d, c}}-\frac{1}{\sqrt{\left\|\tilde{d}_{k}\right\|^{2}}}\right)}{\left(1+\sqrt{\left\|\tilde{d}_{k}\right\|^{2}}\right)^{3}}
$$

which is always negative for $\left\|\tilde{d}_{k}\right\|^{2} \in(0,1]$ by noting that $E_{p, c}<E_{d, c}$ in WCDMA. ${ }^{2}$ The minimum value of $\beta_{l}^{(\mathrm{AA})}$, which corresponds to the least improvement of MRC over $\mathrm{AA}$, is achieved at $\left\|\tilde{d}_{k}\right\|^{2}=1$, given by

$$
\beta_{l, \min }^{(\mathrm{AA})}=\frac{1}{4}\left(1+\frac{E_{p, c}}{E_{d, c}}\right)\left(1+\frac{E_{d, c}}{E_{p, c}}\right)>1
$$

$\forall E_{p, c}, E_{d, c}$. Therefore, it is obvious that $\beta_{l}^{(\mathrm{AA})}>1$.

Through MSE analysis, it can be shown that MRC-based method outperforms all other three channel estimators which make use of the information from the DPCCH channel, DPDCH channel (in the form of soft decoding information), or both of them. The improvement is conditioned on the power allocated to the two channels as well as the soft decoding statistics. Moreover, (24) shows that MRC-method is more efficient when $\|\tilde{d}\|^{2}$ is small, which is the case as the iterative reception starts. Therefore, the MRC channel estimator can speed up the convergence of the decoding by generating enhanced CSI knowledge based on the imperfect decoding information, a prominent feature that allows successful reception in the presence of severe interference.

\section{Simulations}

In this section, we present simulation results of iterative reception that incorporates channel estimation and multiuser decoding in a turbo fashion, over the channels modelled according to the WCDMA specifications. The two multipath channels considered are listed in Table I, which represent two typical scenarios characterized with low and high mobilities.

\begin{tabular}{|l|c|c|c|}
\hline & Power Profile [dB] & Delay Profile [chips] & Speed [km/h] \\
\hline Channel 2 & {$\left[\begin{array}{lll}0 & 0 & 0\end{array}\right]$} & {$\left[\begin{array}{llll}0 & 4 & 77\end{array}\right]$} & 3 \\
\hline Channel 3 & {$\left[\begin{array}{llll}0-3 & -6 & -9\end{array}\right]$} & {$\left[\begin{array}{llll}0 & 1 & 2 & 3\end{array}\right]$} & 120 \\
\hline \hline
\end{tabular}

TABLE I

WCDMA MULTIPATH FADING CHANNEL POWER AND DELAY PROFILES

\footnotetext{
${ }^{2}$ In this paper, we assume $E_{p, c}$ is $6 \mathrm{~dB}$ less than $E_{d, c}$.
} 
In Figure 2, We show the BER performance of the iterative detection which applies AA method to channel estimation for a slow-moving channel. Single user system assumes no MAI, and improves the CSI knowledge by fed back soft symbols over iterations. Its performance at iteration six (in dash circled line) is around $2 \mathrm{~dB}$ away from that for an AWGN channel at $\mathrm{BER}=10^{-3}$. Performance curves of different iterations are also included for a fully loaded CDMA system where $K=$ 32 users are present. It is shown the BER curve at the first iteration flattens out as the signal-to-noise ratio becomes large, due to the presence of severe MAI. The situation improves as the iterative reception proceeds, and the performance on the sixth iteration is only $1.5 \mathrm{~dB}$ from that for the single user case.

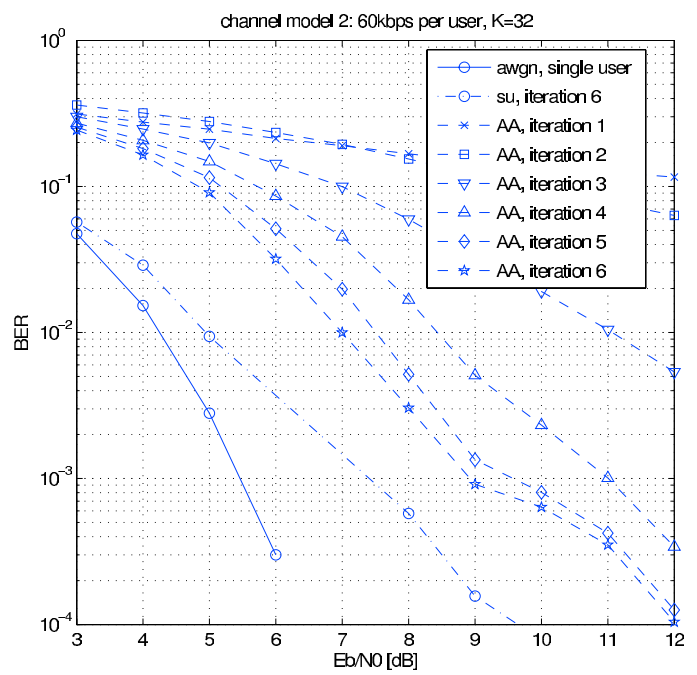

Fig. 2. BER for a fully loaded CDMA system using AA channel estimation over Channel 2

Figure 3 presents the performance of a fully loaded system in a channel characterized with high mobility. Due to the fastvarying nature of the channel, it requires more iterations for the receiver performance to converge. It is shown that the BER at the twelfth iteration is about $3 \mathrm{~dB}$ away from that for single user case for the AA method, while it takes the MRC-based method only eight iterations to achieve the similar performance.

Figure 4 compares the MSE of channel estimates for the MRC estimator to that of the AA method. It is shown that for the first two iterations, MRC-based system gives similar performance to that of the AA-based system since the fed back information from the decoders is very noisy and the channel estimation is determined from the pilot symbol. As the iterations proceed and decoding information becomes more reliable, the improvement of MRC over AA becomes more significant. The MSEs of MRC-based channel estimator stays around $3 \mathrm{~dB}$ below those for AA method, which is larger than the ratio in (25). This can be explained by noting that (25) is the minimum value when both receivers achieve correct decoding, i.e., $\left\|\tilde{d}_{k}\right\|^{2}=1$, while in Figure 4, both systems,

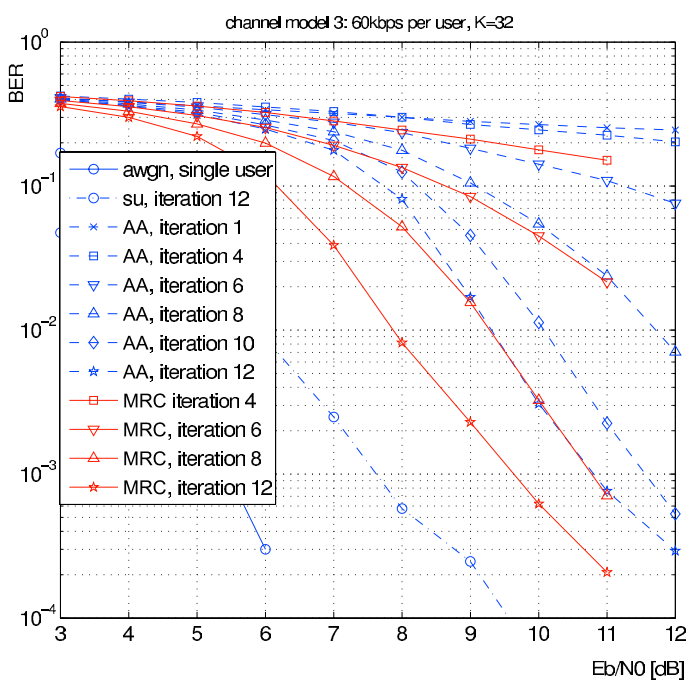

Fig. 3. BER comparison for a fully loaded CDMA system between AA and MRC channel estimation methods over Channel 3

especially the one using AA channel estimation, have some residual errors after iterative decoding.

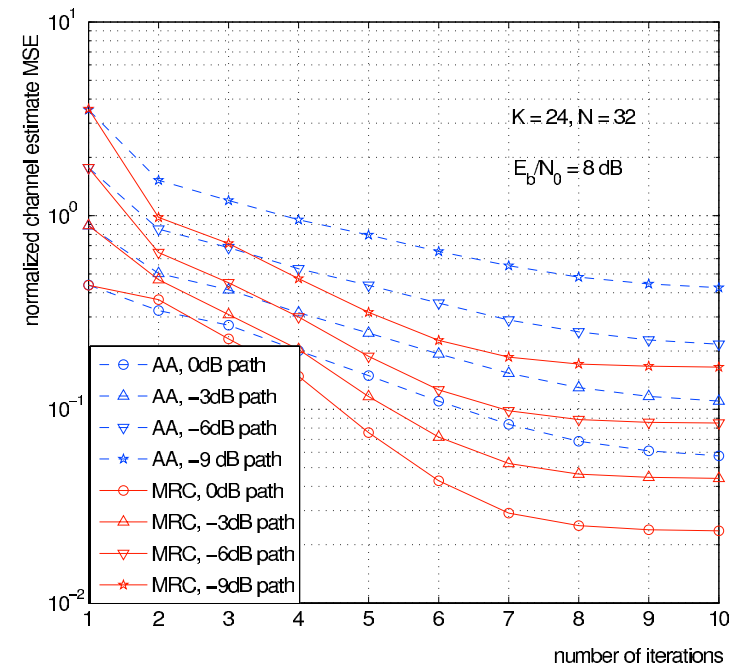

Fig. 4. MSE comparison for a fully loaded CDMA system between AA and MRC channel estimation methods over Channel 3 , at $E_{b} / N_{0}=8 \mathrm{~dB}$.

\section{CONCLUSIONS}

In this paper, we developed an iterative receiver to track the time-varying multipath channel for WCDMA communication systems. The proposed method combines the coarse CSI knowledge based on the soft decoding information and pilot symbols from the DPDCH and DPCCH channels. Under certain assumptions, maximal ratio combining approach can be used to optimize the weights assigned to the DPDCH and DPCCH channel estimates. The optimal weights are subject to the evolving reliability of the soft decoding information 
and the power allocated to the two multiplexed channels. Both MSE analysis and BER simulations show that the MRC-based method outperforms the conventional algorithms.

\section{ACKNOWLEDGEMENT}

Z. Shi and M. C. Reed are with National ICT Australia and affiliated with the Australian National University. National ICT Australia is funded through the Australian Government's backing Australia's Ability initiative and in part through the Australia Research Council.

\section{REFERENCES}

[1] H. Holma and A. Toskala, WCDMA for UMTS, West Sussex, England: Wiley 2002.

[2] P. D. Alexander and A. Grant, "Iterative channel and information sequence estimation in CDMA," IEEE Int. Symp. on Spread-Spectrum Tech. \& Appl., New Jersey, USA, September 2000.

[3] Q. Sun and D. Cox, "Effect of channel estimation error on a new pipelined multi-stage PIC detector for CDMA," IEEE Int. Symp. on Spread-Spectrum Tech. \& Appl., Prague, Czech Republic, September 2002.

[4] A. Lampe, "Iterative multiuser detection with integrated channel estimation for coded DS-CDMA," IEEE Transactions on Communications, pp. 1217-1223, August 2002.

[5] R. Otnes and M. Tüchler, "Iterative channel estimation for turbo equalization of time-varying frequency-selective channels," IEEE Transactions on Wireless Communications, pp. 1918-1923, November 2004.

[6] J. Choi, "Multipath CDMA channel estimation by jointly utilising pilot and traffic channels," IEE Proceedings on Communications, pp. 312-318, October 1999.

[7] S. Bhashyam and B. Aazhang, "Multiuser channel estimation and tracking for long-code CDMA systems," IEEE Transactions on Communications, pp. 1081-1090, July 2002.

[8] C. Schlegel and A. Grant, Coordinated Multiple User Communications, Springer Publishers, 2005.

[9] Z. Shi and C. Schlegel, "Joint iterative decoding of serially concatenated error control coded CDMA", IEEE Journal on Selected Areas in Communications, pp1646-1653, August 2001.

[10] P. D. Alexander, M. C. Reed, J. A. Asenstorfer and C. B. Schlegel, "Iterative multiuser interference reduction: turbo CDMA", IEEE Transactions on Communications, vol. 47, no. 7, pp. 1008-1014, July 1999.

[11] X. Wang and H. V. Poor, "Iterative (turbo) soft interference cancellation and decoding for coded CDMA", IEEE Transactions on Communications, vol. 47, no. 7, pp. 1046-1061, July 1999.

[12] S. Verdu, Multiuser Detection, Cambridge University Press, 1998.

[13] T. A. Summers and S. G. Wilson, "SNR mismatch and online estimation in turbo decoding," IEEE Transactions on Communications, vol. 46, pp. 421-423, April 1998.

[14] D. R. Pauluzzi and N. C. Beaulieu, "A comparison of SNR estimation techniques for the AWGN channel," IEEE Transactions on Communications, vol. 48, pp. 1681-1691, October 2000.

[15] F. Brännström and L. Rasmussen, "Non-data-aided parameter estimation in an additive white Gaussian noise channel," IEEE Int. Symp. on Inform. Theory, Adelaide, Australia, July 2005. 